\title{
Carbon-13 Chemical-Shift Tensors in Indigo: \\ A Two-Dimensional NMR-ROCSA and DFT Study
}

\author{
Sean T. Holmes and Cecil Dybowski \\ Department of Chemistry and Biochemistry, \\ University of Delaware, Newark, Delaware, 19716, USA
}

\begin{abstract}
The principal components of the ${ }^{13} \mathrm{C}$ NMR chemical-shift tensors for the eight unique carbon sites of crystalline indigo have been measured using the ROCSA pulse sequence. The chemical shifts have been assigned unambiguously to their respective nuclear sites through comparison of the experimental data to the results of density-functional calculations employing a refined X-ray diffraction structure. These measurements expand the database of measured aromatic ${ }^{13} \mathrm{C}$ chemical-shift tensors to the indole ring. Magnetic shielding calculations for hypoxanthine and adenosine are also reported. Comparisons of calculations that include the effect of the crystalline lattice with calculations that model indigo as an isolated molecule give an estimate of the intermolecular contribution to the magnetic shielding.
\end{abstract}

Keywords: ${ }^{13} \mathrm{C}$ NMR; recoupling of chemical-shift anisotropy; density-functional theory; indigo; indole; purine; hypoxanthine; adenosine; natural product

Corresponding Author E-mail: dybowski@udel.edu

\section{Introduction}

Indigo, 2,2'-bis(2,3-dihydro-3-oxoindolyliden), is a blue dye with technological, industrial, cultural, and biological significance [1]. The molecular structure and atom-labeling scheme of indigo are given in Fig. 1. Traditionally, indigo has been obtained as a tryptophanderived natural product extracted from tropical plants. The synthesis of indigo has been important in the history of industrial chemistry through processes such as those developed by Baeyer [2-4], including the Baeyer-Drewson reaction [5], and later synthetic routes appropriate to large-scale production by Heumann [6] and by Pfleger [7]. Other than its use as a textile dye, indigo and its derivatives have been used as components of organic semiconductors [8-10].

Nuclear magnetic resonance (NMR) spectroscopy has been used in combination with computational chemistry to study the interaction between indigo molecules and porous clays [11- 
16]. Complexation of indigo derivatives with model fibers has been the subject of investigation by NMR spectroscopy [17, 18]. Chemical analyses of cultural heritage objects with NMR spectroscopy have detected indigo $[18,19]$. NMR has been used to study the biodegradation of indigo dye and its derivatives [20,21], as well as the biosynthesis of indigo and indigo precursors in plants [22] and bacteria [23].

The ubiquity of indigo makes it an excellent model for the study of indole rings, which are found in such places as the amino acid tryptophan and its derivatives. To our knowledge, ${ }^{13} \mathrm{C}$ chemical-shift tensors have not been reported for carbons in indole rings in any material containing such rings. Careful measurements of the ${ }^{13} \mathrm{C}$ chemical-shift parameters of crystalline indigo are important in understanding the crystalline structure, the molecular structure, and the electronic state of the solid. An analysis of structural effects on the ${ }^{13} \mathrm{C}$ NMR parameters, particularly the chemical shifts, of indigo may be of significance in the interpretation of NMR data on indole-containing materials like tryptophan.

The link between chemical shifts and crystal structure is established through quantumchemical calculations such as those based on density-functional theory (DFT). In this work, experimental ${ }^{13} \mathrm{C}$ chemical-shift tensors for all sites in indigo are assigned to their respective lattice sites by comparison to computed values based on a refined X-ray diffraction structure. We also perform calculations on the purine derivative hypoxanthine, and the purine nucleoside adenosine, both of which are structurally similar to indigo (Fig. 1).

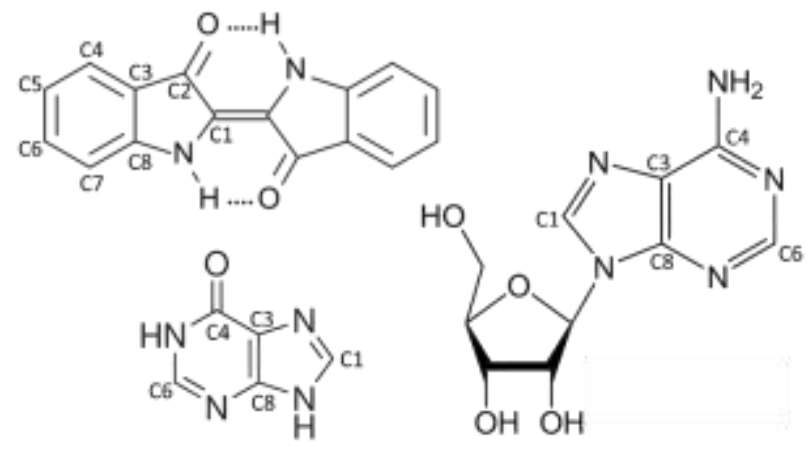

Fig. 1. Structures of indigo (upper left), hypoxanthine (lower left), and adenosine (right). The labeling schemes for the aromatic carbons sites are shown.

\section{Experimental Methods}

Measurements of the ${ }^{13} \mathrm{C}$ chemical-shift tensors in indigo employed the recoupling-ofchemical-shift-anisotropy (ROCSA) sequence, a two-dimensional magic-angle-spinning (MAS) technique in which rotor-synchronized pulse sequences are used to obtain the recoupled powder patterns [26]. The ROCSA sequence is amenable to high-frequency MAS because the recoupling field is required to be only about four times the MAS frequency. The technique was originally developed for the study of isotopically labeled proteins; however, ROCSA and related 
sequences have been applied in the analysis of organic systems other than peptides [27-30]. High-power decoupling of ${ }^{1} \mathrm{H}$ spins used a ${ }^{1} \mathrm{H} 90^{\circ}$ pulse of $2.97 \mu$ s (SPINAL-64 at $100 \mathrm{kHz}$ ). The ROCSA sequence was rotor-synchronized with a MAS frequency of $10.0 \mathrm{kHz} .32 t_{1}$ points, with 544 scans each, were collected with a $6.0 \mathrm{~s}$ recycle delay. The isotropic chemical shifts were externally referenced to tetramethylsilane (TMS) using the amide carbon in $\alpha$-glycine (176.5 ppm) as a secondary reference [31].

Principal components were extracted from the recoupled chemical-shift powder patterns using a numerical simulation with WSOLIDS [32], which identified the principal components of the chemical-shift tensors by searching for singularities in the derivative spectra. The numerical simulations introduced an expansion factor of 0.272, as suggested by Chan and Tycko [26]. The fitted three principal components of the chemical-shift tensor are also reported using Mason's convention [33], the average of the principal components $\delta_{\text {ave }}$, the span $\Omega$, and the skew $\kappa$, through the following relations:

$$
\begin{aligned}
& \delta_{\text {ave }}=\frac{1}{3}\left(\delta_{11}+\delta_{22}+\delta_{33}\right), \\
& \Omega=\delta_{11}-\delta_{33}, \\
& \kappa=\frac{3\left(\delta_{22}-\delta_{\text {iso }}\right)}{\Omega} .
\end{aligned}
$$

The isotropic chemical shift, $\delta_{\text {iso, }}$, was determined in a separate 1D CP/MAS experiment. Within experimental uncertainty, $\delta_{\text {iso }}$ and $\delta_{\text {ave }}$ are the same.

\section{Computational Methods}

Predicted chemical-shift tensors were derived from DFT calculations on molecular clusters representing structures of the three solids $[34,35]$. The models of indigo, hypoxanthine, and adenosine were built from thirteen, eleven, and fifteen molecules, respectively (Fig. 2), to represent the local lattice structure around a single molecule, in accordance with the symmetryadapted-cluster (SAC) model [34]. The cluster models were based on single-crystal diffraction parameters [36-38]. Before first-principles NMR parameters were calculated, the atomic coordinates were optimized, because diffraction-based structures generally give unrealistic hydrogen-atom positions [24, 25, 34]. Optimization was performed using the cluster model with the PBE functional [39] and the cc-pVDZ basis set [40]. For adenosine, the positions of only the hydrogen atoms were refined, as the structure was obtained from neutron diffraction. For indigo and hypoxanthine, the optimizations were performed in two steps where the first optimization allowed only the positions of hydrogen atoms were allowed to relax and the second optimization allowed the entire molecule was allowed to relax. The optimization procedure did not alter the structure of the molecule significantly, with changes in bond lengths being limited to ranges found in previous studies [41]. Nonetheless, such small refinements to the structure are known to improve the quality of magnetic-shielding calculations in many cases [24, 25, 34, 41, 42].

Magnetic shielding was calculated with the gauge-including-atomic-orbital (GIAO) method [43-45] using the $\tau$-dependent hybrid exchange-correlation (XC) functional TPSSh [46]. 
This model chemistry was selected to provide a more rigorous description of electron correlation in the heteroatomic ring. The cluster was partitioned into two layers where the central molecule was given a locally-dense basis set (cc-pVTZ) and the peripheral molecules were given a lessdense basis set (cc-pVDZ). Previous work on organic systems has shown that treating the central molecule of a cluster with a triple- $\zeta$ basis set with additional polarization functions and the outlying molecules with a smaller double- $\zeta$ basis set yields reliable results [34]. Calculations were also performed on single refined molecules taken in isolation, as a means to examine intermolecular effects on the magnetic shielding.

Calculated magnetic-shielding values were converted to chemical shifts through the relationship:

$$
\delta_{\text {calc }}=\frac{\sigma-\sigma_{r e f}}{m},
$$

where $\sigma$ is the calculated magnetic shielding, $\delta_{\text {calc }}$ is the calculated chemical shift, $\sigma_{r e f}$ is the shielding of tetramethylsilane (TMS), and $m$ is an adjustable parameter chosen to remove systematic deviations associated with DFT. The parameters $\sigma_{\text {ref }}(185.4 \mathrm{ppm})$ and $m(-1.012)$ were obtained from a least-squares analysis of a correlation plot between calculated magneticshielding principal components and experimental chemical-shift principal components. All calculations in this study were performed using GAUSSIAN 09 revision B.01 [47].

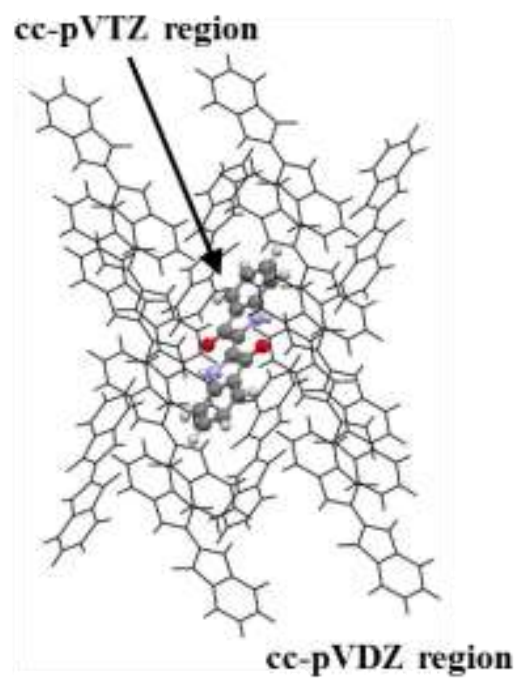

Fig. 2. Crystal packing diagram of indigo (polymorph A, $P{ }_{1} / C$ space group) showing the partition into two layers. The central molecule (cc-pVTZ basis set) is shown in ball-and-stick representation and the peripheral molecules (cc-pVDZ basis set) are shown in a wireframe representation. The peripheral molecules present in the model clusters were chosen according to the symmetry-adapted cluster method. 


\section{Results and Discussion}

NMR Measurements and Peak Assignments. Indigo belongs to the $P 2_{1} / c$ space group with an asymmetric unit composed of half a molecule. The experimental NMR spectrum consists of eight unique resonances located between $112.5 \mathrm{ppm}$ and $187.8 \mathrm{ppm}$, in agreement with expectation based on the crystal structure. Three peaks fall between $118.6 \mathrm{ppm}$ and 121.0 ppm but are resolvable, as shown in the 2D ROCSA spectrum in Fig. 3(a). Subspectra from the ROCSA experiment are shown in Fig. 3(b) and a summary of the ${ }^{13} \mathrm{C}$ chemical-shift tensors extracted from the powder patterns is given in Table 1. Each site exhibits considerable chemicalshift anisotropy with the narrowest powder pattern having a span of $124 \mathrm{ppm}$ and the widest 200 ppm.

An assignment of the resonances is accomplished by correlating the experimental principal components of the ${ }^{13} \mathrm{C}$ chemical-shift tensors with those obtained from DFT calculations (Fig. 4). On this plot are shown the results for indigo, as well as those for hypoxanthine and adenosine (Supporting Information, Tables S1 and S2). Large deviations between experiment and calculation (up to $13 \mathrm{ppm}$ for an individual principal component in indigo) are observed for some sites (Table 1). However, these deviations are well within the range of errors typically reported for aromatic systems containing heteroatoms, as is supported by the calculations on hypoxanthine and adenosine, as well as previous work [41]. This overall assignment yields a root-mean-square $(r m s)$ error between calculation and experiment of 7.1 ppm.

\section{Table 1}

Principal components of the ${ }^{13} \mathrm{C}$ chemical-shift tensors of indigo, with the average of the principal components, isotropic chemical shift, span, and skew. ${ }^{\text {a }}$

\begin{tabular}{cllll||lll}
\hline \hline Site & $\delta_{11}(\mathrm{ppm})^{\mathrm{b}}$ & $\delta_{22}(\mathrm{ppm})^{\mathrm{b}}$ & $\delta_{33}(\mathrm{ppm})^{\mathrm{b}}$ & $\delta_{\text {ave }}(\mathrm{ppm})^{\mathrm{c}}$ & $\delta_{i s o}(\mathrm{ppm})^{\mathrm{c}}$ & $\Omega(\mathrm{ppm})$ & $\kappa$ \\
\hline $\mathrm{C} 1$ & $\mathbf{1 7 3}$ & $\mathbf{1 4 0}$ & $\mathbf{4 9}$ & $\mathbf{1 2 0 . 7} \pm \mathbf{1 . 2}$ & $\mathbf{1 2 1 . 0} \pm \mathbf{0 . 5}$ & $\mathbf{1 2 4} \pm \mathbf{3}$ & $\mathbf{0 . 4 6} \pm \mathbf{0 . 0 6}$ \\
& 177.1 & 144.2 & 48.7 & - & 123.3 & 128.4 & 0.488 \\
\hline $\mathrm{C} 2$ & $\mathbf{2 5 3}$ & $\mathbf{2 1 1}$ & $\mathbf{1 0 0}$ & $\mathbf{1 8 8 . 0} \pm \mathbf{1 . 2}$ & $\mathbf{1 8 7 . 8} \pm \mathbf{0 . 5}$ & $\mathbf{1 5 3} \pm \mathbf{3}$ & $\mathbf{0 . 4 5} \pm \mathbf{0 . 0 5}$ \\
& 255.6 & 202.9 & 102.6 & - & 187.0 & 153.0 & 0.312 \\
\hline $\mathrm{C} 3$ & $\mathbf{1 8 4}$ & $\mathbf{1 4 2}$ & $\mathbf{3 4}$ & $\mathbf{1 2 0 . 0} \pm \mathbf{1 . 2}$ & $\mathbf{1 1 9 . 9} \pm \mathbf{0 . 5}$ & $\mathbf{1 5 0} \pm \mathbf{3}$ & $\mathbf{0 . 4 4} \pm \mathbf{0 . 0 5}$ \\
& 192.1 & 153.7 & 23.6 & - & 123.2 & 168.5 & 0.543 \\
\hline $\mathrm{C} 4$ & $\mathbf{2 0 8}$ & $\mathbf{1 4 9}$ & $\mathbf{1 6}$ & $\mathbf{1 2 4 . 3} \pm \mathbf{1 . 2}$ & $\mathbf{1 2 4 . 1} \pm \mathbf{0 . 5}$ & $\mathbf{1 9 2} \pm \mathbf{3}$ & $\mathbf{0 . 3 9} \pm \mathbf{0 . 0 4}$ \\
& 218.0 & 162.0 & 4.4 & - & 128.1 & 213.6 & 0.476 \\
\hline $\mathrm{C} 5$ & $\mathbf{2 1 0}$ & $\mathbf{1 3 1}$ & $\mathbf{1 5}$ & $\mathbf{1 1 8 . 7} \pm \mathbf{1 . 2}$ & $\mathbf{1 1 8 . 6} \pm \mathbf{0 . 5}$ & $\mathbf{1 9 4} \pm \mathbf{3}$ & $\mathbf{0 . 2 2} \pm \mathbf{0 . 0 2}$ \\
& 214.4 & 128.4 & 11.1 & - & 117.9 & 203.3 & 0.155 \\
\hline $\mathrm{C} 6$ & $\mathbf{2 2 4}$ & $\mathbf{1 5 4}$ & $\mathbf{2 4}$ & $\mathbf{1 3 4 . 0} \pm \mathbf{1 . 2}$ & $\mathbf{1 3 4 . 1} \pm \mathbf{0 . 5}$ & $\mathbf{2 0 0} \pm \mathbf{3}$ & $\mathbf{0 . 3 0} \pm \mathbf{0 . 0 4}$ \\
& 236.5 & 143.9 & 13.4 & - & 131.3 & 223.1 & 0.169 \\
\hline $\mathrm{C} 7$ & $\mathbf{1 9 1}$ & $\mathbf{1 3 3}$ & $\mathbf{1 3}$ & $\mathbf{1 1 2 . 3} \pm \mathbf{1 . 2}$ & $\mathbf{1 1 2 . 5} \pm \mathbf{0 . 5}$ & $\mathbf{1 7 8} \pm \mathbf{3}$ & $\mathbf{0 . 3 5} \pm \mathbf{0 . 0 4}$ \\
& 193.4 & 143.1 & 14.7 & - & 117.1 & 178.7 & 0.436 \\
\hline C8 & $\mathbf{2 2 3}$ & $\mathbf{1 7 6}$ & $\mathbf{5 8}$ & $\mathbf{1 5 2 . 3} \pm \mathbf{1 . 2}$ & $\mathbf{1 5 2 . 3} \pm \mathbf{0 . 5}$ & $\mathbf{1 6 5} \pm \mathbf{3}$ & $\mathbf{0 . 4 3} \pm \mathbf{0 . 0 4}$ \\
& 222.2 & 182.8 & 50.0 & - & 151.7 & 172.2 & 0.542 \\
\hline \hline
\end{tabular}

${ }^{\mathrm{a}}$ Experimental values are shown in bold; calculated values are shown in italics.

${ }^{\mathrm{b}}$ The uncertainty in experimental principal components is $\pm 2 \mathrm{ppm}$.

${ }^{\mathrm{c}}$ The average chemical shift, $\delta_{a v e}$, is derived from principal components derived from the ROCSA 
spectrum. The isotropic shift, $\delta_{i s o}$, is determined from a CP/MAS spectrum. Within experimental error, the two are identical.
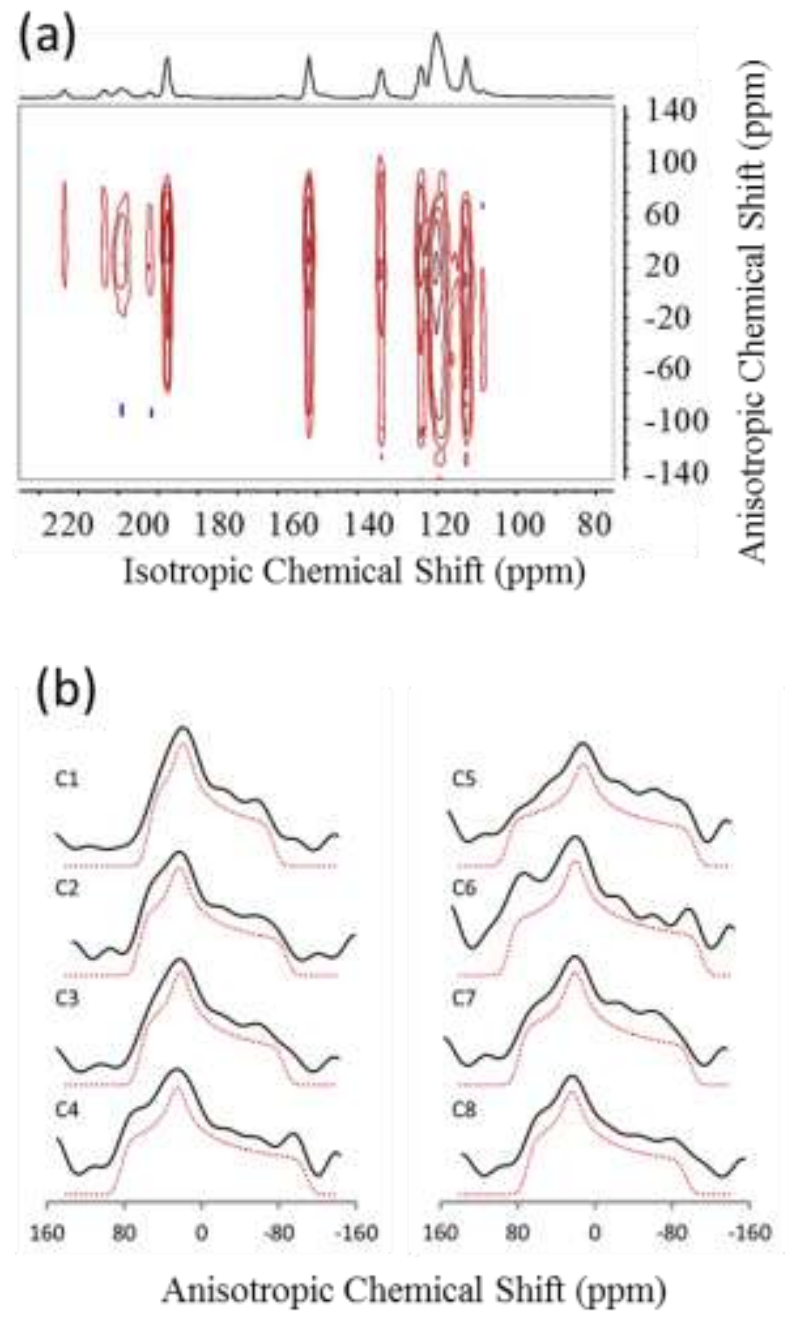

Fig. 3. (a) ${ }^{13} \mathrm{C}$ NMR-ROCSA spectrum of indigo. The chemical-shift scale in the $f 1$ dimension is referenced to the isotropic chemical shift of each resonance, as is customary in ROCSA spectra, whereas the $f 2$ dimension is referenced to TMS. (b) ROCSA chemical-shift subspectra of the eight unique ${ }^{13} \mathrm{C}$ sites in indigo. The upper solid lines are the experimental spectra and the lower traces (dotted lines) are simulated spectra from fitting the chemical-shift powder patterns.

Discussion of ${ }^{13} \mathbf{C}$ Chemical-Shift Tensors of Indigo. Several aromatic ${ }^{13} \mathrm{C}$ chemicalshift tensors of indigo are very different from the tensors of other polycyclic ring systems such as naphthalene [48], indicating the significant effect of incorporating heteroatoms. In sixmembered rings containing a heteroatom, $\pi$-deficient nitrogens have been found to stabilize positive charge at positions ortho and para to the nitrogen [49]. The result of this charge stabilization is that carbons meta to the nitrogen center are more isotropically shielded than carbons ortho and para to the nitrogen. In five-membered heteroatomic rings, carbons one bond from the nitrogen center are typically deshielded relative to carbons two bonds from the nitrogen 
center [49]. C8 of indigo, which resides adjacent to the nitrogen, is the most strongly deshielded carbon site, with $\delta_{i s o}=152.3 \mathrm{ppm}$. C6, three bonds from the nitrogen, is also isotropically deshielded, with $\delta_{i s o}=134.0 \mathrm{ppm}$, as compared to $\mathrm{C} 7$, with $\delta_{i s o}=112.5 \mathrm{ppm}$, which is two bonds from nitrogen. $\mathrm{C} 5$, four bonds from the nitrogen has an intermediate shift of $\delta_{\text {iso }}=118.6$ ppm. Although $\mathrm{C} 1$ is one bond away from the nitrogen, $\delta_{i s o}=120.7 \mathrm{ppm}$, which suggests that its involvement in the double bond compensates the deshielding effects of the adjacent nitrogen.

The aromatic carbon sites in indigo can be parsed into classes based on similarities between the principal components of the ${ }^{13} \mathrm{C}$ chemical-shift tensors. The first major aromatic class is represented by $\mathrm{C} 8$, the carbon site one bond from the nitrogen center. For C8 uniquely, significant deshielding is observed in $\delta_{22}$ and $\delta_{33}$ (176 ppm and $58 \mathrm{ppm}$ from TMS, respectively) relative to the other aromatic carbon sites. The remaining aromatic sites $(\mathrm{C} 3-\mathrm{C} 7)$ can be parsed into classes according to the number of bonds separating them from nitrogen. $\mathrm{C} 3$ and $\mathrm{C} 7$, each two bonds from the nitrogen center, have similar tensors. C4 and C6, each three bonds from nitrogen, form another class. $\mathrm{C} 5$, four bonds from the nitrogen site, is the final class. The principal components of the ${ }^{13} \mathrm{C}$ chemical-shift tensor for $\mathrm{C} 5$ fall within the tolerances of polyclyclic-aromatic hydrocarbons (PAH) reported by Facelli [50]. However, the remaining carbon sites do not fall within the tolerances provided in reference [50], indicating the substantial role of the nitrogen heteroatom on the observed chemical shifts of carbons in the ring.

The class comprised of carbons two bonds from nitrogen can be subdivided into carbons bonded directly to a hydrogen and those which have no directly bonded hydrogen. This difference appears most strongly in the value of $\delta_{33}$. For example, C3, a bridgehead site, is strongly deshielded $\left(\delta_{33}=34 \mathrm{ppm}\right)$ relative to the same principal component of $\mathrm{C} 7\left(\delta_{33}=13\right.$ $\mathrm{ppm}$ ), the latter being bonded to a hydrogen. The remaining aromatic sites in indigo (C4 - C6) are bonded to hydrogens and have $\delta_{33}$ between $15 \mathrm{ppm}$ and $24 \mathrm{ppm}$, similar to that of C7.

Comparison of measured and calculated chemical-shift principal components of indigo to those of hypoxanthine [51] and adenosine [52] gives further insight into the effect of heteroatoms on the carbon chemical-shift tensor. The position of the nitrogen atom in the ring is important to the extended lattice structure because it determines the geometry of intermolecular hydrogen bonds. Of particular interest are $\mathrm{C} 3, \mathrm{C} 6$, and $\mathrm{C} 8$, as these sites are unsubstituted aromatic or bridgehead carbons in all three molecules. The most similar feature of these three molecules is the chemical-shift tensor of C8. In all three molecules, C8 is a bridehead carbon linking a six-membered ring with a five-membered ring one bond from the nitrogen center. In all cases, the principal components fall in the ranges of $223 \pm 2 \mathrm{ppm}, 168 \pm 7 \mathrm{ppm}$, and $59 \pm 1$ for $\delta_{11}, \delta_{22}$, and $\delta_{33}$, respectively. $\mathrm{C} 3$ in indigo is bound to three neighboring carbon atoms, whereas in the purine rings of hypoxanthine and adenosine, $\mathrm{C} 3$ is bonded to two carbon sites and a nitrogen site. For all three molecules, $\delta_{22}$ and $\delta_{33}$ fall in the ranges of $147 \pm 8$ ppm and $42 \pm 7$ ppm, respectively. For indigo, $\delta_{11}$ of C3 is $184 \mathrm{ppm}$ whereas this value is $167 \pm 3 \mathrm{ppm}$ in the purine rings, suggesting that the adjacency to the nitrogen produces shielding of this component. For C6, $\delta_{11}$ and $\delta_{22}$ fall in the ranges of $231 \pm 8 \mathrm{ppm}$ and $154 \pm 5$, respectively, for all three materials. However, $\delta_{33}$ is $24 \mathrm{ppm}$ for indigo, but $61 \pm 5 \mathrm{ppm}$ in the purine rings. It appears that an indole derivative can be distinguished readily from a derivative of purine through analysis of the principal components of the ${ }^{13} \mathrm{C}$ chemical-shift tensors, particularly the $\delta_{33}$ component. 


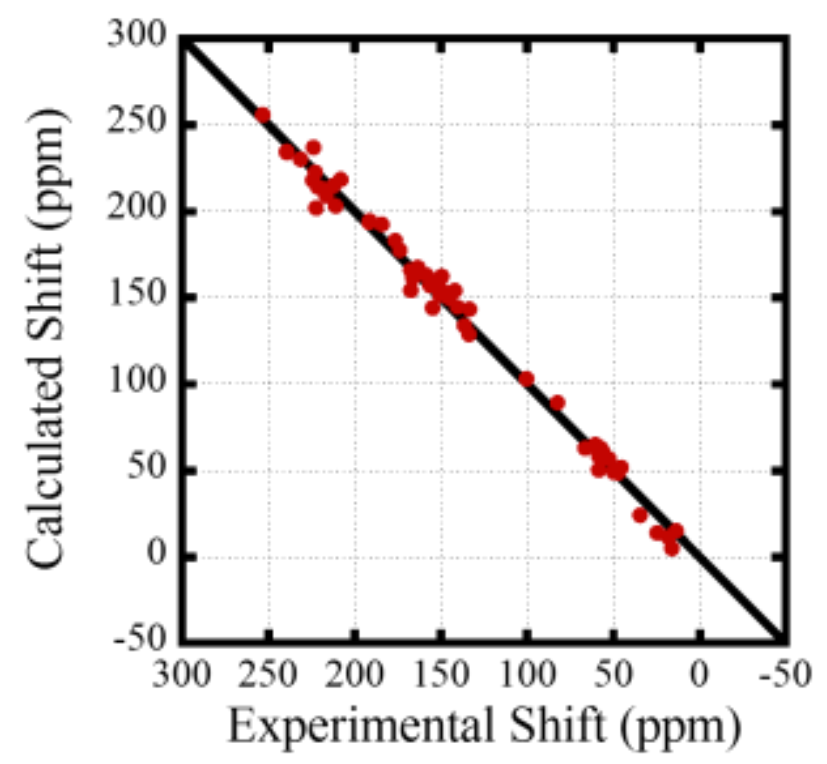

Fig. 4. Correlation of calculated principal chemical-shift components and experimental principal chemical-shift components for ${ }^{13} \mathrm{C}$ sites in indigo, hypoxanthine, and adenosine. Calculations were performed at the TPSSh/cc-pVTZ level. Results for the ribose carbons of adenosine are not included.

Assessment of Lattice Effects. The effect of the crystalline lattice on the calculated chemical-shift tensors in indigo can be assessed by comparison of calculations including the effects of the lattice (as done above) with the results of calculations where lattice effects are ignored, specifically calculations on an isolated indigo molecule, as if it were in the gas phase. The differences in the computed principal components between the two models are given in Table 2.

In indigo, intermolecular interactions result in increased shielding of the ${ }^{13} \mathrm{C}$ principal component $\delta_{33}$ at each carbon site. The average increase is $4.7 \mathrm{ppm}$, with a maximum of 9.2 ppm observed for the keto carbon (C2). The largest effect for any principal component is seen for $\delta_{22}$ of $\mathrm{C} 7$, which is deshielded by $20.2 \mathrm{ppm}$ relative to the isolated molecule. Significant deshielding is seen in the $\delta_{22}$ principal components of $\mathrm{C} 1(10.7 \mathrm{ppm})$ and $\mathrm{C} 4(11.8 \mathrm{ppm})$.

A simple explanation for the differences in chemical shifts between the cluster models and the isolated-molecule models is the existence of weak intermolecular interactions such as $\mathrm{N}$ $\mathrm{H} \cdots \mathrm{O}$ and $\mathrm{C}-\mathrm{H} \cdots \mathrm{O}$ hydrogen bonding that would not be present in an isolated molecule. Süsse suggests that indigo contains a bifurcated $\mathrm{N}-\mathrm{H} \cdots \mathrm{O}$ hydrogen bond where the position of the amine hydrogen is stabilized through intramolecular and intermolecular interactions with nearby keto oxygens [36]. The importance of this interaction is evidenced by the large change in all three principal components of the ${ }^{13} \mathrm{C}$ chemical-shift tensor of $\mathrm{C} 2$ (the keto carbon). The $\mathrm{C}$ $\mathrm{H} \cdots \mathrm{O}$ interaction is known to be important in the crystal packing of purines [53], and appears to be present in indigo as well. For C7 of indigo, the nearest-neighbor atom in the crystal lattice is 
a keto oxygen site, which resides only $2.39 \AA$ from the hydrogen site of C7, a distance less than the sum of the van der Waals radii of the atoms $(2.6 \AA)$. For $\mathrm{C} 4$, the nearest neighbor is also a keto oxygen at a distance of only $2.41 \AA$ from the bonded hydrogen. The difference in the calculated chemical-shift components, we therefore suggest, is an indication of these weak intermolecular $\mathrm{C}-\mathrm{H} \cdots \mathrm{O}$ hydrogen bonds.

The intermolecular contributions to ${ }^{13} \mathrm{C}$ magnetic shielding in the purine rings is very different from that observed in indigo, reflecting the different lattice environments arising from hydrogen bonding. The complete sets of calculated components of hypoxanthine and adenosine are reported in the Supporting Information (Table S3). In hypoxanthine, the largest difference is again observed for the keto carbon, $\mathrm{C} 4$, where $\delta_{11}$ of the site in the cluster is strongly shielded by $20.4 \mathrm{ppm}$ relative to the isolated molecule and $\delta_{22}$ is deshielded by $37.4 \mathrm{ppm}$. These values are consistent with strong intermolecular $\mathrm{N}-\mathrm{H} \cdots \mathrm{O}$ hydrogen bonding in the crystalline material [34]. $\delta_{22}$ for aromatic carbons $\mathrm{C} 1$ and C6 in the cluster are deshielded by $30.4 \mathrm{ppm}$ and $18.1 \mathrm{ppm}$, respectively, relative to the isolated molecule. The effect of intermolecular hydrogen bonding on computed ${ }^{13} \mathrm{C}$ NMR parameters in hypoxanthine, again, is consistent with previous studies [51]. In adenosine, the largest difference is observed in calculated ${ }^{13} \mathrm{C}$ chemical shifts for $\delta_{22}$ of C6, which is deshielded by $17.3 \mathrm{ppm}$ relative to the same site in an isolated molecule. Earlier computational studies have ascribed the large differences in adenosine to the effects of hydrogen bonding [52].

The rms deviation between values calculated for an isolated molecule and the experimental results is $10.6 \mathrm{ppm}$. When the model includes intermolecular effects, as for the cluster calculations, this rms deviation between calculated and experimental values is $7.1 \mathrm{ppm}$. This difference indicates that the inclusion of intermolecular interactions in magnetic-shielding calculations clearly improves the agreement between calculated and experimental results.

Table 2

Differences in calculated chemical shifts between an a molecule of indigo in a cluster and a molecule in isolation. ${ }^{\mathrm{a}}$

\begin{tabular}{cccc}
\hline \hline Site & $\Delta \delta_{11}(\mathrm{ppm})$ & $\Delta \delta_{22}(\mathrm{ppm})$ & $\Delta \delta_{33}(\mathrm{ppm})$ \\
\hline $\mathrm{C} 1$ & 5.4 & 10.7 & -7.8 \\
$\mathrm{C} 2$ & -5.4 & 9.8 & -9.2 \\
$\mathrm{C} 3$ & 3.2 & 7.8 & -2.7 \\
$\mathrm{C} 4$ & -1.7 & 12.1 & -3.9 \\
$\mathrm{C} 5$ & -2.5 & -1.2 & -3.2 \\
$\mathrm{C} 6$ & -0.2 & -6 & -1.1 \\
$\mathrm{C} 7$ & 3.9 & 20.2 & -4.9 \\
$\mathrm{C} 8$ & -5.5 & 4 & -5 \\
\hline${ }^{\mathrm{a} D i f f e r e n c e s}$ & in principal & values & are \\
$\Delta \delta_{j j}=\delta_{j j}^{\text {cluster }}-\delta_{j j}^{\text {isolated }}, j=1,2,3$.
\end{tabular}

\section{Conclusion}


The ${ }^{13} \mathrm{C}$ NMR chemical-shift tensors of the eight carbons of crystalline indigo have been measured with a recoupling-of-chemical-shift-anisotropy (ROCSA) experiment. Theoretical NMR parameters derived from a refined diffraction structure were used as an aid to assign the experimental measurements to their respective sites. Comparison of the principal components of the chemical-shift tensors was necessary, in some cases, to complete the assignments. The assignment is unambiguous and results in an average error of $7.1 \mathrm{ppm}$, a value consistent with materials containing heteroatomic aromatic rings. The principal components of the chemicalshift tensors of the aromatic sites in indigo deviate substantially from those reported for aromatic hydrocarbons such as naphthalene, indicating the significant effect of heteroatoms on the magnetic shielding at carbon sites.

A comparison of the principal components of the ${ }^{13} \mathrm{C}$ chemical-shift tensors of indigo with those of two purine derivatives confirms that magnetic shielding is very sensitive to the position of nitrogen in the ring. To explain the effects on chemical shifts of the presence of other molecules, we posit that the presence of a heteroatom allows indigo to form hydrogen bonds with adjacent molecules in the crystal lattice. Ignoring these intermolecular effects in calculations shows substantial disagreement of the calculated chemical-shift principal components with experiment, with deviations as high as $20.2 \mathrm{ppm}$ for some principal components when lattice effects are ignored, whereas inclusion of these effects through a cluster model brings the experimental and calculated values into good agreement.

\section{Acknowledgements}

C.D. acknowledges the support of the National Science Foundation under Grant CHE0956006. The Delaware COBRE program's NMR facility, supported by a grant from the National Institute of General Medical Sciences - NIGMS (1 P30 GM110758-01) from the National Institutes of Health, is acknowledged. Discussions with Mr. Fahri Alkan, Professor Shi Bai, Professor Robbie Iuliucci, and Dr. Guangjin Hou helped clarify results.

\section{References}

[1] J.M. Matthews, The industrial development of indigo, J. Frankl. Inst., 154 (1902) 423-429.

[2] A. Baeyer, Synthese des isatins und des indigblaus, Chem. Ber., 11 (1878) 1228.

[3] A. Baeyer, Synthese des indigblaus, Chem. Ber., 11 (1878) 1296-1297.

[4] A. Baeyer, Ueber die beziehungen der zimmtsäure zu der indigogruppe, Chem. Ber., 13 (1880) 22542263.

[5] A. Baeyer, V. Drewsen, Darstellung von indigblau aus orthonitrobenzaldehyd, Chem. Ber., 15 (1882) 2856-2864.

[6] K. Heumann, Neue synthesen des indigos und verwandter farbstoffe, Chem. Ber., 23 (1890) 30433045.

[7] J. Pfleger, Process of making indoxyl derivatives, Deutsche Gold \& Silber Scheide Anstalt Vorm. Roessler, Germany . 1901.

[8] M. Irimia-Vladu, E.D. Głowacki, P.A. Troshin, G. Schwabegger, L. Leonat, D.K. Susarova, O. Krystal, M. Ullah, Y. Kanbur, M.A. Bodea, V.F. Razumov, H. Sitter, S. Bauer, N.S. Sariciftci, Indigo - A natural pigment 
for high performance ambipolar organic field effect transistors and circuits, Adv. Mater., 24 (2012) 375380.

[9] B. He, A.B. Pun, D. Zherebetskyy, Y. Liu, F. Liu, L.M. Klivansky, A.M. McGough, B.A. Zhang, K. Lo, T.P. Russell, L.W. Wang, New form of an old natural dye: Bay-annulated indigo (BAI) as an excellent electron accepting unit for high performance organic semiconductors, J. Am. Chem. Soc., 136 (2014) 1509315101.

[10] O. Pitayatanakul, T. Higashino, T. Kadoya, M. Tanaka, H. Kojima, M. Ashizawa, T. Kawamoto, H. Matsumoto, K. Ishikawa, T. Mori, High performance ambipolar organic field-effect transistors based on indigo derivatives, J. Mater. Chem. C, 2 (2014) 9311-9317.

[11] S. Ovarlez, F. Giulieri, A.M. Chaze, F. Delamare, J. Raya, J. Hirschinger, The incorporation of indigo molecules in sepiolite tunnels, Chem. Eur. J., 15 (2009) 11326-11332.

[12] R. Giustetto, F. Xamena, G. Ricchiardi, S. Bordiga, A. Damin, R. Gobetto, M.R. Chierotti, Maya blue: A computational and spectroscopic study, J. Phys. Chem. B, 109 (2005) 19360-19368.

[13] E. Lima, A. Guzman, M. Vera, J.L. Rivera, J. Fraissard, Aged natural and synthetic Maya blue-like pigments: What difference does it make?, J. Phys. Chem. C, 116 (2012) 4556-4563.

[14] J. Raya, J. Hirschinger, S. Ovarlez, F. Giulieri, A.M. Chaze, F. Delamare, Insertion of indigo molecules in the sepiolite structure as evidenced by ${ }^{1} \mathrm{H}^{29} \mathrm{Si}$ heteronuclear correlation spectroscopy, Phys. Chem. Chem. Phys., 12 (2010) 14508-14514.

[15] A. Domenech, M.T. Domenech-Carbo, M.S. del Rio, S. Goberna, E. Lima, Evidence of topological indigo/dehydroindigo isomers in Maya blue-like complexes prepared from palygorskite and sepiolite, J. Phys. Chem. C, 113 (2009) 12118-12131.

[16] B. Hubbard, W.X. Kuang, A. Moser, G.A. Facey, C. Detellier, Structural study of Maya Blue: Textural, thermal and solid-state multinuclear magnetic resonance characterization of the palygorskite-indigo and sepiolite-indigo adducts, Clays Clay Miner., 51 (2003) 318-326.

[17] C. Bonechi, S. Martini, C. Rossi, Interaction study of indigo carmine with albumin and dextran by NMR relaxation, Journal of Materials Science, 46 (2011) 2541-2547.

[18] R.C. Hoffman, R.C. Zilber, R.E. Hoffman, NMR spectroscopic study of the Murex trunculus dyeing process, Magn. Reson. Chem., 48 (2010) 892-895.

[19] N. Proietti, F. Presciutti, V. Di Tullio, B. Doherty, A.M. Marinelli, B. Provinciali, N. Macchioni, D. Capitani, C. Miliani, Unilateral NMR, ${ }^{13} \mathrm{C}$ CPMAS NMR spectroscopy and micro-analytical techniques for studying the materials and state of conservation of an ancient Egyptian wooden sarcophagus, Anal. Bioanal. Chem., 399 (2011) 3117-3131.

[20] M. Ramya, B. Anusha, S. Kalavathy, Decolorization and biodegradation of indigo carmine by a textile soil isolate Paenibacillus larvae, Biodegradation, 19 (2008) 283-291.

[21] H. Podgornik, I. Poljansek, A. Perdih, Transformation of indigo carmine by Phanerochaete chrysosporium ligninolytic enzymes, Enzyme Microb. Technol., 29 (2001) 166-172.

[22] Z.Q. Xia, M.H. Zenk, Biosynthesis of indigo precursors in higher plants, Phytochemistry, 31 (1992) 2695-2697.

[23] S. Hart, K.R. Koch, D.R. Woods, Identification of indigo-related pigments produced by Escherichia coli containing a cloned rhodococcus gene, J. Gen. Microbiol., 138 (1992) 211-216.

[24] F. Liu, C.G. Phung, D.W. Alderman, D.M. Grant, Carbon-13 chemical shift tensors in methyl glycosides, comparing diffraction and optimized structures with single-crystal NMR, J. Am. Chem. Soc., 118 (1996) 10629-10634.

[25] F. Liu, A.M. Orendt, D.W. Alderman, D.M. Grant, Carbon-13 chemical shift tensors in pentaerythritol, J. Am. Chem. Soc., 119 (1997) 8981-8984.

[26] J.C.C. Chan, R. Tycko, Recoupling of chemical shift anisotropies in solid-state NMR under high-speed magic-angle spinning and in uniformly ${ }^{13} \mathrm{C}$-labeled systems, J. Chem. Phys., 118 (2003) 8378-8389. 
[27] S. Paramasivam, A. Balakrishnan, O. Dmitrenko, A. Godert, T.P. Begley, F. Jordan, T. Polenova, Solidstate NMR and density functional theory studies of ionization states of thiamin, J. Phys. Chem. B, 115 (2011) 730-736.

[28] L.B. Casabianca, M.A. Shaibat, W.W.W. Cai, S. Park, R. Piner, R.S. Ruoff, Y. Ishii, NMR-based structural modeling of graphite oxide using multidimensional ${ }^{13} \mathrm{C}$ solid-state NMR and ab initio chemical shift calculations, J. Am. Chem. Soc., 132 (2010) 5672-5676.

[29] G. Hou, I.-J.L. Byeon, J. Ahn, A.M. Gronenborn, T. Polenova, Recoupling of chemical shift anisotropy by R-symmetry sequences in magic angle spinning NMR spectroscopy, J. Chem. Phys., 137 (2012) 134201.

[30] R.M. Orr, M.J. Duer, Recoupling of chemical-shift anisotropy powder patterns in MAS NMR, J. Magn. Reson., 181 (2006) 1-8.

[31] V.G. Malkin, O.L. Malkina, D.R. Salahub, Influence of intermolecular interactions on the ${ }^{13} \mathrm{C}$ NMR shielding tensor in solid $\alpha$-glycine, J. Am. Chem. Soc., 117 (1995) 3294-3295.

[32] K. Eichele, HBA 3.1 and WSOLIDS, University of Tubingen, Tubingen, 2013.

[33] J. Mason, Conventions for the reporting of nuclear magnetic shielding (or shift) tensors suggested by participants in the Nato Arw on NMR shielding constants at the University-of-Maryland, College-Park, July 1992, Solid State Nucl. Magn. Reson., 2 (1993) 285-288.

[34] S.T. Holmes, R.J. Iuliucci, K.T. Mueller, C. Dybowski, Density functional investigation of intermolecular effects on ${ }^{13} \mathrm{C}$ NMR chemical-shielding tensors modeled with molecular clusters, J. Chem. Phys., 141 (2014) 164121.

[35] F. Alkan, C. Dybowski, Calculation of chemical-shift tensors of heavy nuclei: A DFT/ZORA investigation of ${ }^{199} \mathrm{Hg}$ chemical-shift tensors in solids, and the effects of cluster size and electronic-state approximations, Phys. Chem. Chem. Phys., 16 (2014) 14298-14308.

[36] P. Susse, M. Steins, V. Kupcik, Indigo: Crystal structure refinement based on synchrotron data, Z. Kristallogr., 184 (1988) 269-273.

[37] H.W. Schmalle, G. Hanggi, E. Dubler, Structure of hypoxanthine, Acta Crystallogr., Sect. C, 44 (1988) 732-736.

[38] W.T. Klooster, J.R. Ruble, B.M. Craven, R.K. McMullan, Structure and thermal vibrations of adenosine from neutron diffraction data at 123 K, Acta Crystallogr., Sect. B, B47 (1991) 376-383.

[39] J.P. Perdew, K. Burke, M. Ernzerhof, Generalized gradient approximation made simple, Phys. Rev. Lett., 77 (1996) 3865-3868.

[40] T.H. Dunning, Gaussian basis sets for use in correlated molecular calculations. I. The atoms boron through neon and hydrogen, J. Chem. Phys., 90 (1989) 1007-1023.

[41] J.K. Harper, R. Iuliucci, M. Gruber, K. Kalakewich, Refining crystal structures with experimental ${ }^{13} \mathrm{C}$ NMR shift tensors and lattice-including electronic structure methods, CrystEngComm, 15 (2013) 86938704.

[42] J.C. Johnston, R.J. Iuliucci, J.C. Facelli, G. Fitzgerald, K.T. Mueller, Intermolecular shielding contributions studied by modeling the ${ }^{13} \mathrm{C}$ chemical-shift tensors of organic single crystals with plane waves, J. Chem. Phys., 131 (2009) 144503/144501-144503/144511.

[43] J.R. Cheeseman, G.W. Trucks, T.A. Keith, M.J. Frisch, A comparison of models for calculating nuclear magnetic resonance shielding tensors, J. Chem. Phys., 104 (1996) 5497-5509.

[44] G. Schreckenbach, T. Ziegler, Calculation of NMR shielding tensors using gauge-including atomic orbitals and modern density functional theory, J. Phys. Chem., 99 (1995) 606-611.

[45] R. Ditchfield, Self-consistent perturbation theory of diamagnetism, Mol. Phys., 27 (1974) 789-807.

[46] J.M. Tao, J.P. Perdew, V.N. Staroverov, G.E. Scuseria, Climbing the density functional ladder: Nonempirical meta-generalized gradient approximation designed for molecules and solids, Phys. Rev. Lett., 91 (2003). 
[47] M.J. Frisch, G.W. Trucks, H.B. Schlegel, G.E. Scuseria, M.A. Robb, J.R. Cheeseman, G. Scalmani, V. Barone, B. Mennucci, G.A. Petersson, H. Nakatsuji, M. Caricato, X. Li, H.P. Hratchian, A.F. Izmaylov, J. Bloino, G. Zheng, J.L. Sonnenberg, M. Hada, M. Ehara, K. Toyota, R. Fukuda, J. Hasegawa, M. Ishida, T. Nakajima, Y. Honda, O. Kitao, H. Nakai, T. Vreven, J.A. Montgomery, J.E. Peralta, F. Ogliaro, M. Bearpark, J.J. Heyd, E. Brothers, K.N. Kudin, V.N. Staroverov, R. Kobayashi, J. Normand, K. Raghavachari, A. Rendell, J.C. Burant, S.S. Iyengar, J. Tomasi, M. Cossi, N. Rega, J.M. Millam, M. Klene, J.E. Knox, J.B. Cross, V. Bakken, C. Adamo, J. Jaramillo, R. Gomperts, R.E. Stratmann, O. Yazyev, A.J. Austin, R. Cammi, C. Pomelli, J.W. Ochterski, R.L. Martin, K. Morokuma, V.G. Zakrzewski, G.A. Voth, P. Salvador, J.J. Dannenberg, S. Dapprich, A.D. Daniels, Farkas, J.B. Foresman, J.V. Ortiz, J. Cioslowski, D.J. Fox, Gaussian 09, Revision B.01, Wallingford CT, 2009.

[48] M.H. Sherwood, J.C. Facelli, D.W. Alderman, D.M. Grant, Carbon-13 chemical shift tensors in polycyclic aromatic compounds. 2. Single-crystal study of naphthalene, J. Am. Chem. Soc., 113 (1991) 750-753.

[49] W.W. Paudler, Nuclear Magnetic Resonance: General Concepts and Applications, John Wiley \& Sons, Ltd., New York, 1988.

[50] J.C. Facelli, B.K. Nakagawa, A.M. Orendt, R.J. Pugmire, Cluster analysis of ${ }^{13} \mathrm{C}$ chemical shift tensor principal values in polycyclic aromatic hydrocarbons, J. Phys. Chem. A, 105 (2001) 7468-7472.

[51] K. Maliňáková, L. Novosadová, M. Lahtinen, E. Kolehmainen, J. Brus, R. Marek, ${ }^{13} \mathrm{C}$ chemical shift tensors in hypoxanthine and 6-mercaptopurine: Effects of substitution, tautomerism, and intermolecular interactions, J. Phys. Chem. A, 114 (2010) 1985-1995.

[52] D. Stueber, D.M. Grant, ${ }^{13} \mathrm{C}$ and ${ }^{15} \mathrm{~N}$ chemical shift tensors in adenosine, guanosine dihydrate, $2^{1}-$ deoxythymidine, and cytidine, J. Am. Chem. Soc., 124 (2002) 10539-10551.

[53] D.J. Sutor, C-H $\cdots$ O hydrogen bonds in crystals, Nature, 195 (1962) 68-\&. 
